\title{
LOS PROFESORES FRENTE A LAS ENFERMEDADES DE LA FRANCIA RURAL DEL SIGLO XIX: INFLUENCIA MÉDICA, AUTOMEDICACIÓN Y PEDAGOGÍA ADAPTADA A LOS CUIDADOS DEL CUERPO ${ }^{1}$
}

Séverine Parayre

Institut Catholique de Paris, ISP-Faculté d'éducation, France Chercheure associée au laboratoire TEC (Techniques et Enjeux du Corps), Paris Descartes severineparayre@gmail.com

\section{RESUMEN}

Basándonos en la primera encuesta-concurso promovida entre los profesores rurales por el ministerio de Instrucción Pública en 1860, proponemos un estudio de las enfermedades en la escuela y la preocupación de los profesores por el cuerpo enfermo. La pregunta planteada a los profesores resulta general: “¿Cuáles son las necesidades de la instrucción primaria en una comunidad rural, bajo el triple punto de vista de la escuela, los alumnos y el maestro?", y deja entrever una influencia de la medicina, la inquietud por la falta de médicos y las problemáticas de salud de los alumnos y de las poblaciones. Presentaremos aquello que revela el corpus en relación con la circulación y la influencia de las ideas médicas así como con las transformaciones en el papel y en las actividades pedagógicas del profesor.

Palabras clave: Profesores. Rural. Salud.

\section{THE TEACHERS FACED WITH DISEASES IN RURAL FRANCE OF THE 19TH CENTURY: MEDICAL INFLUENCE, SELF-MEDICATION AND PEDAGOGIES ADAPTED TO BODY CARE}

\begin{abstract}
We propose a study of diseases in school and the concern of the sick body by the primary school teachers, since first survey-competition established by the ministry of education in 1860 for rural primary school teachers. The question asked to the primary school teachers is general: "what are the needs for the primary education in a countryside, in the triple point of view of school, pupils and teacher?". We look for influence of medicine, concerns of the lack of doctor and the problems of pupils' health. We shall present our corpus' analysis for the circulation of medical ideas and their influence and the change in the teacher's role and educational activities.
\end{abstract}

Keywords: Teachers. Countryside. Health.

\footnotetext{
${ }^{1}$ Artículo traducido por Lucia Martínez Moctezuma.
} 


\title{
OS PROFESSORES FRENTE ÀS ENFERMIDADES NA FRANÇA RURAL DO SÉCULO XIX: INFLUÊNCIA MÉDICA, AUTO-MEDICAÇÃO E PEDAGOGIAS ADAPTADAS AOS CUIDADOS DO CORPO
}

\begin{abstract}
RESUMO
Tendo em vista a primeira pesquisa-concurso realizada pelo ministério de instrução pública em 1860 junto às instituições rurais, nossa proposta é a de estudar as doenças na escola e a preocupação dos profesores com o corpo doente. A questão colocada aos professores é geral: "Quais são as necessidades da instrução primária em uma comunidade rural, segundo o triplo ponto de vista da escola, dos alunos e do mestre?". Esta questão deixa entrever uma influência da medicina, das preocupações com a falta de médicos e das problemáticas de saúde dos alunos e das populações. Nós apresentaremos aquilo que revela o corpus para a circulação das ideias médicas, sua influência e as transformações no papel e nas atividades pedagógicas do educador.
\end{abstract}

Palavras-chave: Educadores. Rural. Saúde.

\section{LES INSTITUTEURS FACE AUX MALADIES DANS LA FRANCE RURALE DU XIXesiècle: INFLUENCE MÉDICALE, AUTOMÉDICATION ET PÉDAGOGIES ADAPTÉES AUX SOINS DU CORPS}

\section{RÉSUMÉ}

Depuis la première enquête-concours établie par le ministère de l'Instruction publique en 1860 auprès des instituteurs ruraux, nous proposons une étude des maladies à l'école et de la préoccupation du corps malade par ces instituteurs. La question posée aux instituteurs est générale: "Quels sont les besoins de l'instruction primaire dans une commune rurale, au triple point de vue de l'école, des élèves et du maître?", et laisse entrevoir une influence de la médecine, des préoccupations du manque de médecin et des problématiques de santé des élèves et des populations. Nous présenterons ce que révèle le corpus pour la circulation des idées médicales et leur influence et les bouleversements dans le rôle et les activités pédagogiques de l'enseignant.

Mots-clés: Enseignants. Rural. Santé.

\section{INTRODUCCIÓN Y PROBLEMÁTICA: LA SALUD EN EL CAMPO VISTA POR LOS PROFESORES}

Mucho antes de la creación en Francia de la higiene escolar ${ }^{2}$ en el curso de la Tercera República (1870-1940), los preceptos de higiene estaban destinados a los profesores y profesoras

\footnotetext{
${ }^{2}$ La higiene escolar tiene por principio difundir la propaganda higienista. Utiliza todos los procedimientos posibles de la publicidad, las películas y los carteles que difunden la pedagogía, etc (cf. Stéphane Frioux y Didier Nourrisson, 2015).
} 
así como a las poblaciones escolares. En efecto, desde la segunda mitad del siglo XVIII, observamos cierta preocupación en relación con los cuidados y la prevención en torno a la salud desarrollada en los establecimientos escolares. Este fenómeno sigue la "revolución médica" que se opera en Francia y en Europa en el curso del Siglo de las Luces (PORTER, 1997, p. 306). Concierne a las escuelas del Antiguo Régimen en dos aspectos: convertir los establecimientos en salubres y preservarlos de las epidemias. En el curso del siglo XIX estas dos inquietudes sanitarias están presentes y evolucionan según las enfermedades y los avances científicos, además de otros intereses higienistas, relacionados con la alimentación y el ejercicio físico (PARAYRE, 2011). Si bien los historiadores de la educación y de la salud han desarrollado estos problemas y continúan enriqueciendo el estudio internacional de la historia de la salud escolar, aún quedan trabajos pendientes (NOURRISSON, 2002, VIGARELLO, 2004; RIONDET et GO, 2013; FRIOUX et NOURRISSON, 2015; RIONDET 2015; PARAYRE, 2017). Las preocupaciones más importantes conciernen las actividades pedagógicas y los procedimientos que se siguieron para lograr el cuidado y el bienestar desarrollados por los maestros y las maestras. En efecto, si bien disponemos de una investigación minuciosa sobre la propaganda higienista (FRIOUX et NOURRISSON, 2015), no podemos distinguir aún cuales fueron los métodos de enseñanza empleados por los profesores y de que manera ellos y ellas contribuyeron a desarrollar un interés por el cuidado personal de los estudiantes y de la escuela coincidiendo con la aparición de nuevas sensibilidades (VIGARELLO, 2014; DELUERMOZ, 2012). Cierto, algunos de ellos siguieron minuciosamente los preceptos modelo de la higiene, pero otros, se mostraron reticentes a semejanza de los maestros Freinet en el siglo XX (RIONDET, 2015). Para aprehender mejor toda la complejidad de la historia de la salud en la escuela, resulta significativo orientar la investigación desde la voz de los pedagogos. Para ello es necesario ocuparnos del conjunto de la profesión y no solamente de aquellos del medio urbano. El medio rural y las practicas de los maestros y maestras quedan aún por explorar.

Así pues, proponemos en este artículo una contribución a esta historia, estudiando las condiciones escolares en el medio rural francés, tomando como base el discurso de los profesores. Se trata de un primer corpus del que disponemos y, que podrá enriquecerse con otros archivos en el transcurso de nuestras investigaciones, para construir una historia de la salud escolar en el medio rural. El corpus estudiado en el presente artículo resulta también valioso en la medida en que los historiadores de la educación que lo han estudiado, no han reparado en las 
preocupaciones que conciernen el cuerpo, la higiene y la salud de los alumnos (JACQUETFRANCILLON, 1999; NICOLAS, 2012; GHERARDHI, 2012).

\section{UN CORPUS INÉDITO Y RICO: LA ENCUESTA-CONCURSO DEL MINISTRO GUSTAVE ROULAND}

\section{Descripción del corpus}

En 1860, Gustave Rouland, ministro francés de Instrucción Pública lanza una encuestaconcurso entre los profesores rurales, con la intención de recabar testimonios que sirvieran para mejorar las escuelas públicas. Por primera vez en la historia de la educación francesa, un ministro inaugura el dialogo con los maestros y solicita su opinión sobre la instrucción. El ministro plantea una pregunta abierta y amplia: “¿Cuáles son las necesidades de la instrucción primaria en una comunidad rural, desde el triple punto de vista de la escuela, los alumnos y el maestro?"3. La encuesta es importante por su complejidad (el conjunto del territorio francés, comprendida Córcega y Argelia), su originalidad (educación popular y educación rural) y su intención, dar la palabra a los maestros, mal considerados y desatendidos por los gobiernos en turno. Los institutores laicos rurales que fueron cuestionados formaban un grupo numeroso ${ }^{4}$, y su opinión fue valorada. Maestros portadores de mejoras sociales, sanitarias y educativas para los estudiantes atrasados del campo $^{5}$, reclamaban asistencia y gratuidad escolar, se oponían al trabajo infantil y a la indiferencia de los padres, con el deseo de formar futuros ciudadanos virtuosos e instruidos. Contamos con un discurso muy detallado de los maestros sobre diversas temáticas de la educación. Un corpus que también puede ser estudiado desde la perspectiva de la salud.

Entre el 15 de diciembre de 1860 y el 3 de febrero de 1861,5940 institutores respondieron y enviaron sus informes, para ser seleccionados por los inspectores de enseñanza primaria y la academia de cada departamento. Constituyen aproximadamente el $16 \%$ del conjunto

\footnotetext{
${ }^{3}$ Arrêté du 12 décembre 1860.

${ }^{4} 33253$ profesores por 7425 profesoras, cf. F. Jacquet-Francillon, Instituteurs avant la République, La profession d'instituteur et ses représentations de la monarchie de Juillet au Second Empire, Villeneuve d'Ascq, Presses Universitaires du Septentrion, 1999, p. 164.

5 “campagnes arriérées" Arch Nat. (Archives Nationales) $\mathrm{F}^{17} 10775$, instituteur Louis-Adolphe Herner, commune de Grougis, Aisne, 27 janvier 1861.
} 
de la profesión. 1207 relatos pasaron la primera selección regional y fueron examinados por los inspectores de las escuelas primarias del departamento de la Sena. Sólo el 3\% del corpus original fue seleccionado ${ }^{6}$. Se trataba de una encuesta-concurso, en el que fueron otorgados algunos premios, lo que evidentemente debió motivar a los maestros, pues el primer premio recibió 1200 francos, equivalente a dos años de salario. ¿Qué ocurrió con los ganadores, con aquello que fueron capaces de recomendar, de sus buenas ideas sobre la educación y sus mejoras, fueron útiles en el terreno escolar? Nos enfrentamos aquí al primer problema: las 1207 memorias seleccionadas no han sido localizadas hasta el día de hoy, quizá están perdidas y por esta razón no podemos saber exactamente por qué fueron seleccionadas y si efectivamente fueron útiles al ministerio y, ¿en qué medida ? Para entender la situación hace falta consultar los testimonios de los sucesores de Rouland ${ }^{7}$. En definitiva son 4733 memorias las que están hoy disponibles en los Archivos Nacionales de París, conservadas en 41 cajas clasificadas por departamentos ${ }^{8}$. Estas memorias fueron rechazadas durante el concurso. Los motivos son varios y figuran en la primera página de cada uno de los documentos, a veces en un tono implacable. Seleccionamos algunos comentarios: "exageración", "desconsuelo y falta de motivación, sin soluciones prácticas", "tratado de pedagogía que no responde a las preguntas", "buenas ideas, inmersas en un mar de palabras con citaciones de varias páginas", "gran pretensión, estilo incorrecto, neologismos que irritan e imágenes incoherentes, muy difusas y gratuitamente filosóficas”9.

Algunos profesores fueron más prolíficos que otros, sus recuerdos se extienden desde una hasta ciento cuarenta y siete páginas como máximo ${ }^{10}$. La mayor parte de los maestros retoman en su texto el orden indicado por el ministerio, dando sus puntos de vista sobre la escuela, los alumnos y el maestro. Después, el contenido de los tres puntos principales varía según las preocupaciones individuales. En la parte consagrada a la escuela se agrupan las preguntas sobre el edificio: disposiciones exteriores e interiores, algunas veces con un plan anexo creado por el maestro, cuestiones de salubridad de los locales y otros. Esta primera parte es rica

\footnotetext{
${ }^{6}$ La lista de los 172 memorias seleccionadas en la etapa final fueron publicadas en el Journal des instituteurs $\mathrm{n}^{\circ} 38$ du 22 septembre 1861 .

${ }^{7}$ No insistiré en este punto que se encuentra publicado en otro texto (PARAYRE, 2011, p. 250).

${ }^{8}$ Las cajas están disponibles en los Archivos Nacionales en el sitio de Pierrefitte en Seine-Saint-Denis, bajo las referencias de $\mathrm{F}^{17}$ 10758-10798: concours ouvert aux instituteurs 1861.

${ }^{9}$ En el orden de las citas: Arch. Nat. $\mathrm{F}^{17}$ 10778, D'Hubert Homobon, commune de Franvillers, Somme, 28 janvier 1861. $F^{17}$ 10787, Marie-Louis Lalire, Châtillon-sur-Marne, Marne, $1^{\text {er }}$ février 1861. $F^{17} 10787$, Alphonse Patoux, Brusson, Marne, 28 janvier 1861. $\mathrm{F}^{17}$ 10787, François Morise, Corrobert, Marne, 23 janvier 1861.

${ }^{10} \mathrm{La}$ memoria más grande del corpus es la de Alphonse Patoux, Brusson, Marne, 28 janvier 1861 (Arch. Nat. $\mathrm{F}^{17}$ 10787) con 147 páginas, es también, la más extensa de los reportes de los maestros de todo el territorio.
} 
en informaciones sobre lo que nosotros definimos como la higiene del espacio, porque nos informa sobre el saneamiento de los locales y la observación sobre diversas instalaciones. Informa también sobre ciertos aprendizajes como los de agricultura y horticultura, ampliamente recomendados por los maestros en un mundo todavía muy rural ( $75 \%$ de la población). La parte consagrada a los alumnos reúne desde la descripción, más o menos detallada, de las disciplinas que se enseñan (lectura, escritura, calculo, religión), hasta cuestiones sobre la gratuidad escolar, el ausentismo (aún frecuente), las relaciones con las familias y los vecinos. Es también en este apartado que algunos maestros hablan de la higiene del cuerpo y el cuidado de la salud. La última parte concierne al maestro, donde encontramos sus compromisos con sus superiores, sus conflictos con el cura o el alcalde y los consejeros municipales. Revelan además sus problemas con el salario, la jubilación y con su propia salud.

\section{METODOLOGÍA EMPLEADA}

Empleamos una metodología mezclando los métodos cuantitativos y cualitativos de manera que pudiéramos tener una visión general y precisa del discurso de los profesores.

En primer lugar, definimos los departamentos: 31 departamentos equivalentes a 2083 memorias que fueron seleccionadas según los siguientes seis criterios (cf. carta 1):

- La tasa de analfabetismo y la evolución de la educación, porque el Nordeste tiene una tasa de analfabetismo más baja. El Sudoeste y Córcega tienen una tasa más fuerte (FURET et OZOUF, 1977; PÉLISSIER y RÉBAUDO, 2004).

- La tasa de medicalización, ciertos departamentos están submedicalizados (menos medicinas y pocos espacios de cuidado) como los departamentos del Oeste de Francia (LÉONARD, 1978).

- La naturaleza del territorio, algunas regiones son más áridas, mineras o arboladas. Esto tendrá un impacto significativo en los problemas y los cuidados de la salud, las enfermedades y las dolencias pueden variar.

- Los departamentos limitados por otros países, con la idea de percibir o no las influencias externas a la Francia. Como en el caso de Pas-de-Calais, el Norte, Haut-Rhin y Bas-Rhin, los Hautes-Alpes.

- El número de memorias por departamento. La prioridad fueron los departamentos con una alta tasa de memorias (Pas-de-Calais con 264 memorias, Seine-Inferior con 229 memorias) y los 
departamentos, valiosos por su ubicación pero con pequeña tasa de memorias (Argelia con 27 memorias, Vendée con 28 memorias).

- Los medios de comunicación, porque algunas regiones estaban todavía desprovistas de todo sistema ferroviario, y por lo tanto con menor posibilidades de desplazamientos. Que es todavía el caso en 1860 de los departamentos al Oeste y al Sudoeste, sin conexión con el tren (CARON, 1997).

Después de esta primera selección, notamos que era necesario estudiar el conjunto de las memorias de cada uno de los departamentos seleccionados, porque el discurso de la salud no está presente en todos los profesores, de lo contrario corríamos el riesgo de perder datos muy importantes.

Carta 1: Departamentos seleccionados (en negro los elegidos para el estudio además de Córcega y Argelia, no indicados en este mapa).

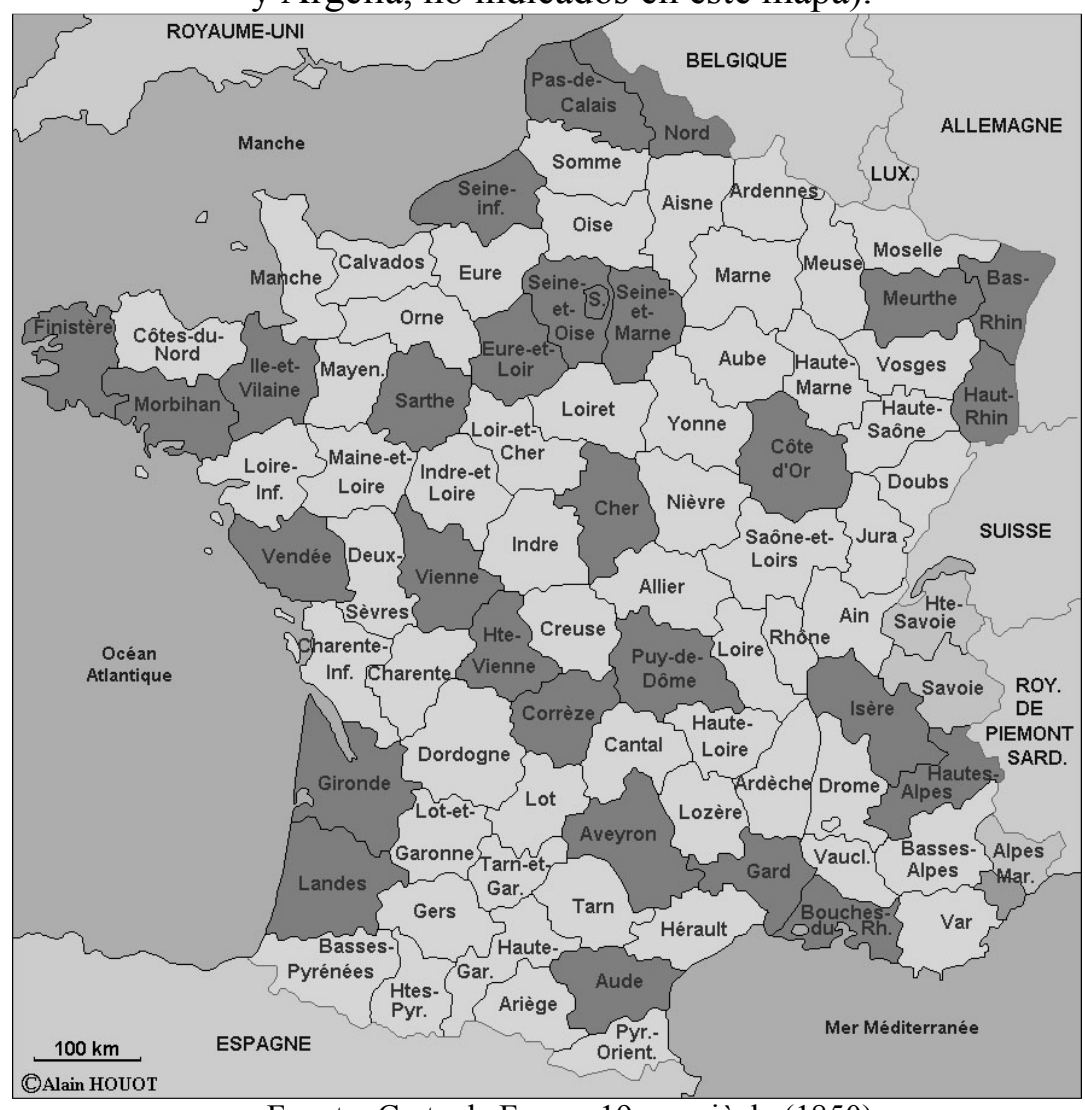

Fuente: Carte de France 19eme siècle (1850).

A continuación, se procedió a elaborar una base de datos (con Access, versión 2007). De acuerdo con nuestros cuestionamientos sobre las preocupaciones de los profesores en el tema de 
la salud (ámbitos de la prevención y el cuidado) y la composición de todo el corpus, fueron elaborados 21 cuadros: 8 con información general, 4 con datos sobre los lugares y espacios escolares, 6 más específicos con cuestiones sobre la prevención y 3 con cuestiones sobre el cuidado de la salud y los procedimientos médicos seguidos por los maestros (cf. Figura 1). Cada uno de los cuadros puede incluir 3 o hasta 19 ítems de información específica, según el discurso de los maestros. Nuestra codificación cambió también en la medida en que añadimos datos e hicimos el análisis, nos percatamos de los ajustes que hacía falta por hacer. Algunos de los discursos de los maestros fueron objeto de un estudio más detallado porque se referían específicamente a la cuestión del cuidado de la salud.

De esta manera pudimos analizar muchos perfiles de profesores, desde aquellos que nunca evocaron el tema de la salud hasta quienes comenzaron a dar su opinión y recomendaron tratamientos específicos sobre los males y las enfermedades de los alumnos.

Figura 1. Detalles de los 21 cuadros elaborados a partir de la base de datos.

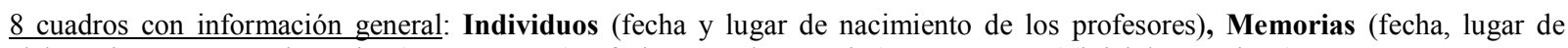
elaboración y número de páginas), Profesión (profesión actual y anterior), Formación ( (inicial y continua), Señalamiento en las memorias (de los inspectores de la escuela primaria, de la Academia y de la región del Sena), Problemas anexos (ligados a la escolaridad: a la gratuidad escolar, a la asistencia en escuelas privadas y los vinculados con la función del maestro: de la jubilación a la baja retribución), Escuelas (situación geográfica, número y sexo de los alumnos), Relaciones (con los padres de familia, la autoridad municipal, el cura, los superiores, los habitantes).

4 cuadros específicamente vinculados con el lugar y el espacio escolar: Higiene del lugar (todas las cuestiones de salubridad interior: de la humedad a la calefacción), Exterior de la escuela (todos los servicios: el patio de recreo, el pozo, etc), Crítica local (críticas hechas en común, los arquitectos), Mobiliario escolar (estado y solicitud de mobiliario).

6 cuadros específicos sobre la prevención: Modificación del régimen de vida (pausa, juegos, comodidades), Higiene del cuerpo (limpieza, piojos, inspección, educación corporal, alimentación), Ejercicio de los alumnos (físicos, militares, calidad), Pedagogía (lecciones de higiene, agricultura, horticultura, metas de la escuela, moral, calidad del maestro, pedagogía por el ejemplo, curso para adultos y curso de ciencias), Vestido (limpieza, calzado), Corrección del cuerpo (vigilancia de la postura, castigo, trabajo).

3 cuadros específicos sobre el cuidado de la salud: Salud del maestro (si habla, calidad), Enfermedades (tipo, vacunas), Intervenciones (naturaleza de la enfermedad y los involucrados: medico, profesor, otros).

Fuente: Elaboración del autor. 


\section{RESULTADOS: EL MAESTRO RURAL FRENTE A LA SALUD DE LOS ALUMNOS}

\section{De la influencia médica de la salud en los maestros rurales}

\section{Una influencia médica menor e importante vinculada a la prevención más que al cuidado}

A pesar del gran número de temas evocados por estos profesores y sus ideas progresistas, faltan referencias a la salud. En efecto, sólo 6\% de éstos habla de la atención a la salud (visita al médico, enfermedades, cuidados, vacuna). Es mayor el número de profesores que habla de la prevención (educación corporal, limpieza del cuerpo, ejercicios físicos, alimentación), el porcentaje se eleva hasta un $20 \%$ en algunos departamentos (sobretodo en el Norte de Francia). Pero en el tema de la salud no hay unanimidad dentro de la profesión y en algunos departamentos incluso esta ausente (en el Oeste de Francia en toda la región de Bretaña). Hay que señalar que hasta este periodo de 1860-1861 el movimiento higienista desarrollado desde los años 1830 favoreció sobre todo los preceptos ligados a la prevención, en parte porque los médicos no habían aún determinado la etiología de las enfermedades y se mostraban en desacuerdo con la enfermedad y su origen. Desde los años 1830, el gobierno francés, consciente del papel primordial de los profesores de educación primaria contribuyó, de igual manera, a difundir entre ellos una consciencia sanitaria orientada hacia los cuidados de la salud (PARAYRE, 2010). Sin embargo, resulta obvio no encontrar un discurso medico dominante entre los maestros, porque en ese momento ellos no consideraban esta función de cuidado y de prevención como un elemento esencial de su profesión y de su pedagogía. Es importante señalar también que la enseñanza de la higiene no es aún obligatoria en la formación de los profesores y que la mayor parte procura documentarse de manera personal para instruirse en el tema de la higiene. Esta enseñanza, aún facultativa, se volverá obligatoria en la formación de los maestros hasta $1865^{11}$.

\footnotetext{
${ }^{11}$ Programmes officiels de l'enseignement secondaire spécial avec les instructions ministérielles et autres documents officiels (relatifs à l'exécution de la loi du 21 juin 1865), Paris, Ch. Delagrave, 1866.
} 


\section{Una influencia médica especifica, delimitada y educativa}

La mayor parte de los maestros que toca el tema de la salud hace referencia a los preceptos médicos de su época. Muchos de sus comentarios sugieren que se documentaron, o que estuvieron en contacto con un médico y por tanto, están influidos por el discurso de la época, a semejanza de este profesor de la Meurthe (Nordeste de la Francia) quien precisaba que si él: “conociera un poco de higiene, sería capaz de prestar servicio especialmente en el campo donde la ignorancia y la lejanía del médico compiten contra la salud de los hombres"12. O este profesor de Haut-Rhin (Nordeste de Francia) quien recuerda que la sensibilidad de la población rural hacia los cuidados todavía no estaba presente y que la confianza en la medicina científica no estaba asentada tampoco en el campo:

En su enfermedad [el pequeño cultivador], en lugar de llamar o consultar a un médico, que le ocasionaría nuevos gastos, prefiere emplear los remedios que le aconseja un vecino o una buena mujer. No es sino hasta el último momento cuando la enfermedad se ha enraizado, y todos los otros medios han fallado, que recurre a un experto ${ }^{13}$.

Las preocupaciones de los profesores son también desiguales de un departamento a otro. El discurso de la salud esta completamente ausente en algunos departamentos, que son también los que están submedicados (faltan medicamentos y lugares apropiados) como en el caso del Oeste (Norte y Sur). Son también los departamentos con una fuerte tasa de analfabetismo (FURET y OZOUF, 1977; PÉLISSIER y RÉBAUDO, 2004), donde los profesores están más preocupados por el aprendizaje de la lengua. Así, existen prioridades educativas geográficamente localizadas y diferentes según el contexto regional. Por ejemplo, resulta significativo descubrir que en Bretaña (Oeste de la Francia) y en Córcega (Isla al Sureste de Francia), el discurso de los maestros está mucho mas centrado en el aprendizaje del francés, debido a las lenguas regionales que predominan. Esto resulta significativo para este maestro del Finistere (Extremo Oeste de Francia, cf. carta 1) quien muestra su descontento así: "La masa del pueblo ignora lo que es leer y

\footnotetext{
12 “connaît un peu d'hygiène, il sera à même de rendre bien des services, surtout dans les campagnes où l'ignorance et l'éloignement du médecin concourent contre la santé des hommes" Arch. Nat. $\mathrm{F}^{17} 10783$ bobine 3, Charles Béguet, Villacourt, Meurthe, 20 janvier 1861.

13 “Dans sa maladie [le petit cultivateur], au lieu d'appeler ou de consulter un médecin qui lui occasionnerait de nouveaux frais, il préfère employer les remèdes que lui conseille un voisin ou une bonne femme. Ce n'est qu'à la dernière extrémité quand la maladie a pris racine, et que tous les autres moyens ont échoué qu'il a recours à un homme de l'art"Arch. Nat. $\mathrm{F}^{17}$ 10794, Guttmauer Léger, Dessenheim, Haut-Rhin, 28 janvier 1861.
} 
escribir, cinco de seis niños no han asistido a la escuela" ${ }^{\text {" }}$. A medida que estos maestros pueden resolver el aprendizaje elemental, ausente en la mayor parte de los estudiantes, pueden pensar y señalar otros aspectos, como los de la salud, que de hecho aparece de manera secundaria en el corpus, incluso de manera velada.

Comparando a los maestros que evocan la salud frente a quienes no lo hacen, descubrimos que aquellos que hablan y dan su opinión, son aquellos que consideran a su profesión como la de un educador y por tanto diversifican sus funciones. Por el contrario, quienes no hablan nunca de la salud son aquellos que están más orientados a la enseñanza de las disciplinas y consideran su función no como la de un educador, sino más bien limitada a la instrucción, cuya función se centra principalmente en el aprendizaje de habilidades básicas de lectura, escritura y cálculo.

Podemos decir entonces que este corpus ayuda a comprender la existencia de diferentes categorías de profesores con sensibilidades variables, no todos comparten las mismas consideraciones en torno a la infancia, el bienestar o la educación corporal. Algunos piensan que ser profesor es ocuparse de la educación y por tanto de "formar el cuerpo, el espíritu y el corazón" ${ }^{15}$. En este caso podemos entender que el cuerpo y sus cuidados pudieran estar entre sus prioridades educativas $\mathrm{y}$, por tanto, estarían preocupados por la educación corporal. A semejanza de este maestro quien confirma su importancia: "Parece, de entrada, que el maestro, no teniendo a sus alumnos bajo su vigilancia inmediata más que una pequeña parte de la jornada, no tiene más que ocuparse de cuidar el desarrollo de varias partes de su cuerpo, sería un gran error pensarlo así"16.

\section{Cuidar, vigilar y atender}

La gráfica 1 muestra los procedimientos de cuidado y salud señalados por los profesores en tres departamentos, Pas-de-Calais, el Norte (Norte de Francia) y el Finistère (Noroeste de Francia). El Pas-de-Calais y el Norte constituyen los dos departamentos en los cuales los

\footnotetext{
14 "La masse du peuple ignore ce que c'est lire et écrire, cinq sur six au moins des enfants n'ont pas fréquenté l'école"Arch. Nat. F ${ }^{17} 10792$ Bobine 4 et 5 Victorien-Marie Le Louarn, Trégunc, Finistère, 15 janvier 1861.

15 “former le corps, l'esprit et le cœur” Arch. Nat. F1 ${ }^{7}$ 10791, Pierre Sinturat, Saint-Benoît, Vienne, $1^{\text {er }}$ février 1861.

16 "Il semble au premier abord que le maître, n'ayant ses élèves sous sa surveillance immédiate qu'une faible partie de la journée, n'a que peu à s'occuper du développement des diverses parties de leur corps, ce serait une grande erreur de le penser" Arch. Nat. F ${ }^{17} 10777$, Jean-Baptiste Boutemy, Tortequesne, Pas-de-Calais, 31 janvier 1861.
} 
maestros parecen estar más implicados en la salud. En el Norte, refieren al médico por las visitas, los cuidados y la atención regular hacia la salud de los niños. Consideran sobre todo la importancia de la prevención y la atención, pero dejan el cuidado a cargo del médico. Por el contrario, en Pas-de-Calais, el médico no es requerido y es el profesor quien se considera apto para cuidar, vigilar y atender. En el seno de los departamentos aledaños, puede haber incluso diferentes referencias e implicaciones variables, no habiendo todavía un llamado sistemático al médico. En estos mismos departamentos (Norte y Pas-de-Calais) los maestros señalan su interés por atender la salud pero dejan la responsabilidad al médico para atender las enfermedades y dolencias más importantes y no curables (cf. gráfica 2). De esta manera el médico tendría a su cargo los accidentes mas graves, las epidemias y los problemas de la piel cuando el maestro no pudiera tratarlos o no fueran de su competencia. Esto implica por otra parte la intervención del maestro a pesar de tener o no la habilidad para ello empleando remedios médicos y no médicos como lo veremos más adelante. En el Finistère por el contrario, el maestro no se preocupa por tomar estas precauciones y prefiere enviar al niño enfermo a su casa (cf. gráfica 1). Parece no haber necesidad de involucrarse en los problemas de salud de los estudiantes.

Gráfica 1: Acciones de salud señaladas por el profesor.

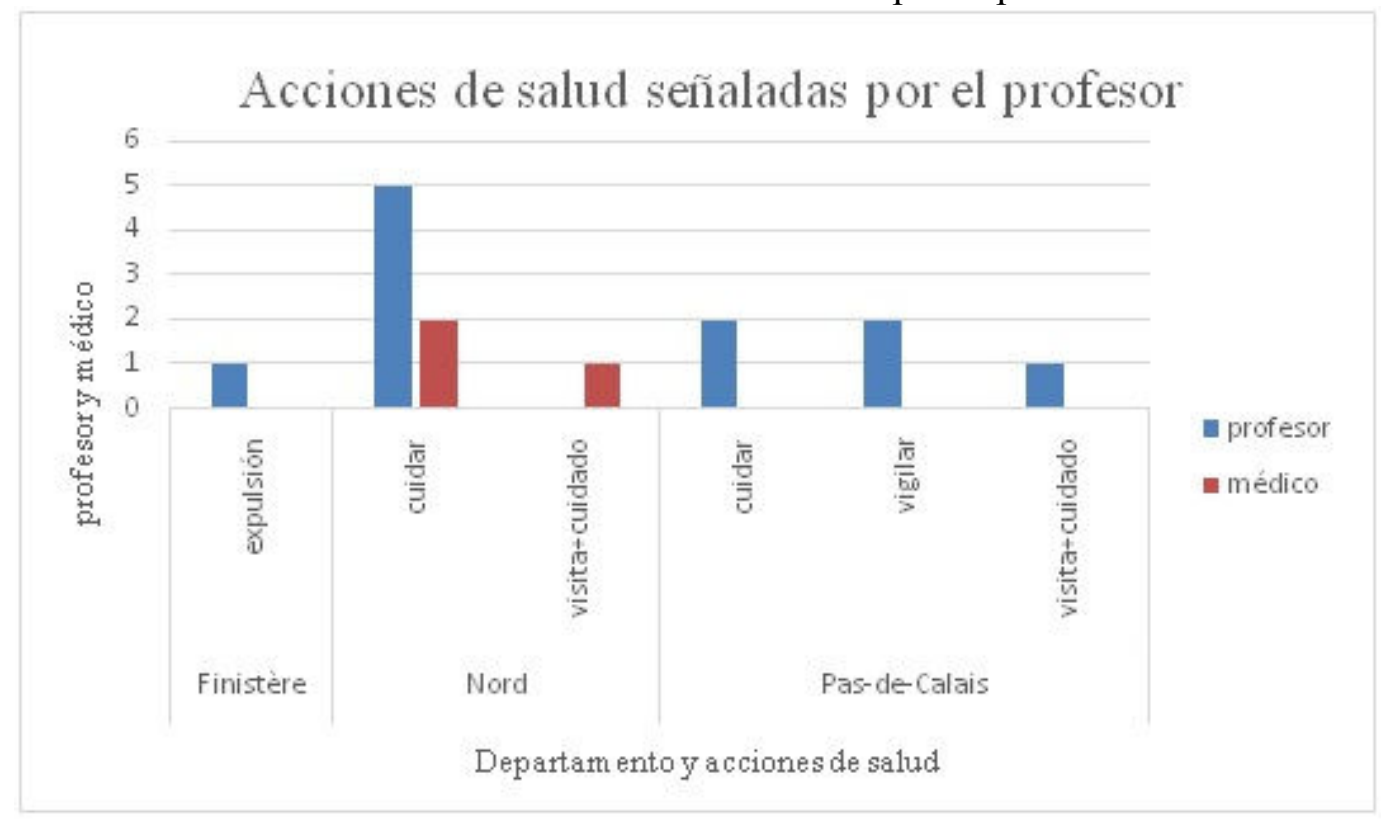

Fuente: Elaboración del autor.

Finistère: Noroeste de Francia n: 46

Nord, Pas-de Calais: Nordeste de Francia Nord: 161; Pas-de-Calais: 245; n: 406

Finistère, Nord, Pas-de-Calais n: 452 
Gráfica 2: Acciones de salud por enfermedades y malestares (departamentos del Norte y del Pasde-Calais).

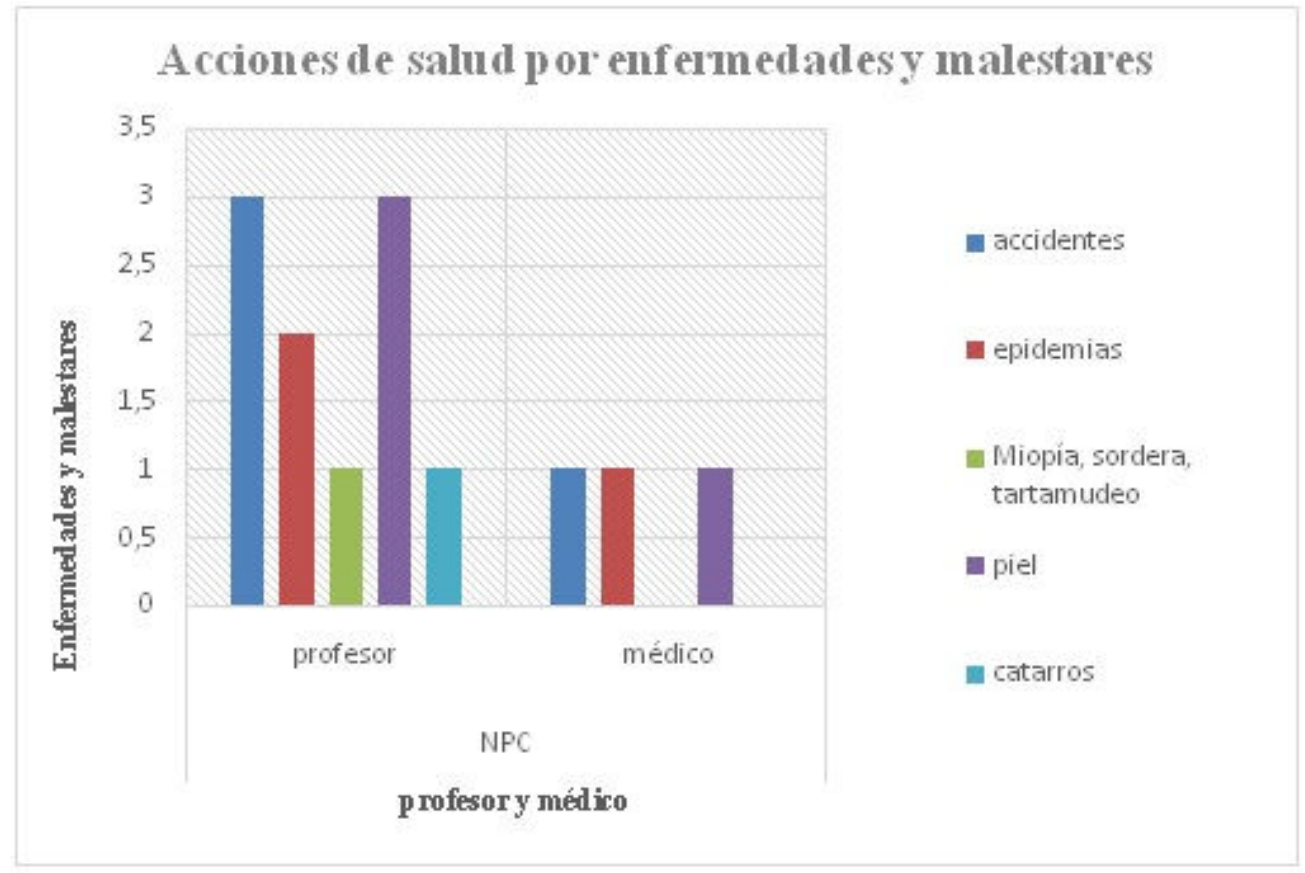

Fuente: Elaboración del autor.

NPC: significa los departamentos del Norte y de Pas-de-Calais (Nordeste de Francia) n: 406

\section{De la automedicación en la nuevas generaciones de profesores}

\section{Los remedios sin referencia médica}

Los maestros que evocan la salud y la atención, muestran en sus discursos un alejamiento médico tanto físico (ubicados lejos de los médicos), como intelectual (no siempre refieren a verdaderos cuidados médicos). Sus discursos reflejan desconocimiento frente a diferentes males y enfermedades y encontramos soluciones médicas que están relacionadas con simples remedios de la vida diaria. A veces los profesores no parecen documentarse en obras médicas y refieren a concepciones y logros personales en el cuidado del cuerpo. El hecho de que no hayan sido formados en estos preceptos médicos e higiénicos y que no hayan estado nunca en contacto con la comunidad médica puede explicar de alguna manera sus decisiones profanas y personales. Librados a ellos mismos en las alejadas zonas rurales, se ven obligados a ejercer la automedicación y a mezclar las instrucciones de la medicina científica, que pudieron haber leído 
o retenido después de haber visitado a un médico, con los remedios profanos producto de su propia experiencia y trayectoria de vida. Por ejemplo, en Audresselles, pequeña localidad de Pasde-Calais (Norte de Francia) el maestro atento al cuidado de la salud, que juzga como primordial, expresa diversos consejos para las caídas, los esguinces, el sangrado de nariz, las lombrices en los intestinos, los cuerpos extraños en el oído y en el ojo, los defectos de los órganos vocales. Hace indicaciones generales que no toman necesariamente en cuenta los avances médicos de la época, y que se reducen a simples remedios. Al señalar que para "mitigar el dolor de un esguince" y precisar que "hay que aplicar agua fría en la inflamación" 17 , se queda en una práctica común, un gesto instintivo, y no documentado ${ }^{18}$, no hace referencia a la medicina científica, aunque afirma que para las cuestiones de gravedad sería necesario consultar al médico. Los cuidados de salud no resultan fáciles si no hay una formación previa para aquellos que son inexpertos, tampoco podemos entender que algunos profesores no intervinieran, o lo hicieran de manera restringida, en la prevención más sencilla, la que no requiere de un gran conocimiento de la anatomía. Por otra parte administran lo que podemos considerar como primeros auxilios aun si su objetivo no fuera para nada sustituir al médico. Cada uno tiene entonces la posibilidad de elegir o no una intervención para proteger la salud de los estudiantes. Pero en ausencia del personal médico en el campo, es fácil entender que un gran número de vecinos y maestros rurales utilizaran remedios, que se usaban regularmente y que no corresponden, necesariamente, a los de la medicina científica.

\section{Los remedios con referencia medica}

Otros, sin embargo, manifiestan en sus discursos un acercamiento con el médico local y declaran inspirarse de sus tratamientos, incluso queriendo reemplazarlo en caso de ausencia. Por ejemplo, el maestro Vasseur en Selles en el Norte de Francia señala la oportunidad que tiene de utilizar el remedio del médico Vlemincka para tratar a niños con sarna. Le parece de utilidad tener algunas nociones médicas, especialmente ante la carencia de médicos en las zonas rurales. Para este profesor la atención debe ser gratuita y los "médicos de caridad podrían venir y ayudar

\footnotetext{
${ }^{17}$ Arch. Nat. $\mathrm{F}^{17}$ 10777, François Marie Augustin Baude, Audresselles, Pas-de-Calais, 30 janvier 1861.

${ }^{18} \mathrm{Si}$ leemos las obras médicas de la época sobre esta cuestion, los remedios parecen mucho más elaborados, cf. Encyclographie des sciences médicales, Répertoire générale des sciences médicales au XIXe siècle Volumes 25 à 26 , Bruxelles, Hauman et Cie, 1843, p. 165-166.
} 
con sus luces y su competencia con visitas trimestrales o semestrales a las escuelas y más a menudo si fuera necesario"19. Una medicina cantonal y una sociedad de ayuda mutua son también reivindicadas en el Este de Francia, porque a menudo el maestro, un altruista, puede aceptar pagar, con su propio dinero, la visita del médico ${ }^{20}$. El maestro comprometido considera que puede aceptar otros compromisos y ampliar de la misma manera sus funciones iniciales de educación para la prevención:

\begin{abstract}
He escuchado algunas veces, decir a los médicos que harían bien en encomendar a los maestros la propagación de la vacuna, que no requiere de un cirujano experto, al mismo tiempo que podríamos hacer un seguimiento de estas operaciones con un médico responsable. La autoridad podría evaluar si existen serios inconvenientes para darnos esta nueva atribución. ${ }^{21}$.
\end{abstract}

Este importante testimonio revela también una incipiente colaboración, posible y reivindicada entre el médico y el profesor de la escuela primaria en la difusión de la medicina preventiva. Nos encontramos así en las primicias del trabajo en común de dos oficios orientados en los cuidados del cuerpo y la salud.

\title{
Una conciencia sanitaria entre los más jóvenes profesores
}

Las edades de los maestros que respondieron a la encuesta variaba de 21 a 77 años. Gracias a la base de datos que construimos y comparando los discursos sobre la salud entre las generaciones de maestros, pudimos analizar la importancia de la edad en las implicaciones de los maestros. Para hacerlo, se compararon cinco departamentos: dos departamentos del Noreste, el Pas-de-Calais y el Norte con mayor índice de participación en la salud, con tres departamentos del Noroeste de la Bretaña, Finistère, la Isla y Vilaine y Morbihan, con baja tasa de participación en salud. Esta comparación entre BVP (Noroeste) y PN (Noreste) (cf. gráfica 3) muestra que son las generaciones más jóvenes de 27 a 44 años que tienen un discurso sobre la salud y sus cuidados, el corte de edad de 27 a 31 años reúne de manera reiterada la evocación de las enfermedades y el malestar (accidentes, epidemias, miopía, sordera, tartamudeo, enfermedades de

\footnotetext{
19 "médecins de bienfaisance pourraient venir aider de leurs lumières et de leur concours par des visites trimestrielles ou semestrielles les écoles et plus souvent selon les besoins" Arch. Nat. $F^{17} 10774$, bobine 4, Pierre-Marie-Auguste Vasseur, Selles, Pas-de-Calais, 25 janvier 1861.

${ }^{20}$ Arch. Nat. $\mathrm{F}^{17}$ 10794, Auguste Hagemann, Gundershoffen, Bas-Rhin, 28 janvier 1861.

21 “J'ai entendu quelquefois des médecins dire à ce sujet qu'on ferait bien de confier aux instituteurs la propagation de la vaccine, qui ne demande pas un habile chirurgien, d'autant plus qu'on pourrait faire surveiller ces opérations par un docteur préposé à cet effet. L'autorité pourrait examiner s'il y a de graves inconvénients à nous donner cette nouvelle attribution Arch. Nat. F ${ }^{17}$ 10796, Antoine-Jean Fabre, Prades-d'aubrac, Aveyron, 25 janvier 1861.
}

Rev. Iberoam. Patrim. Histórico-Educativo, Campinas (SP), v. 2, n. 3, p. 14-37, jul./dez. 2016 
la piel y resfriados). Una conciencia sanitaria se va formando entre las jóvenes generaciones más influidas por las ideas y las preocupaciones médicas e higiénicas de su tiempo y más dispuestas a involucrarse en la educación sanitaria corporal.

Gráfica 3: Enfermedades y malestares evocadas según la edad de los de los maestros.

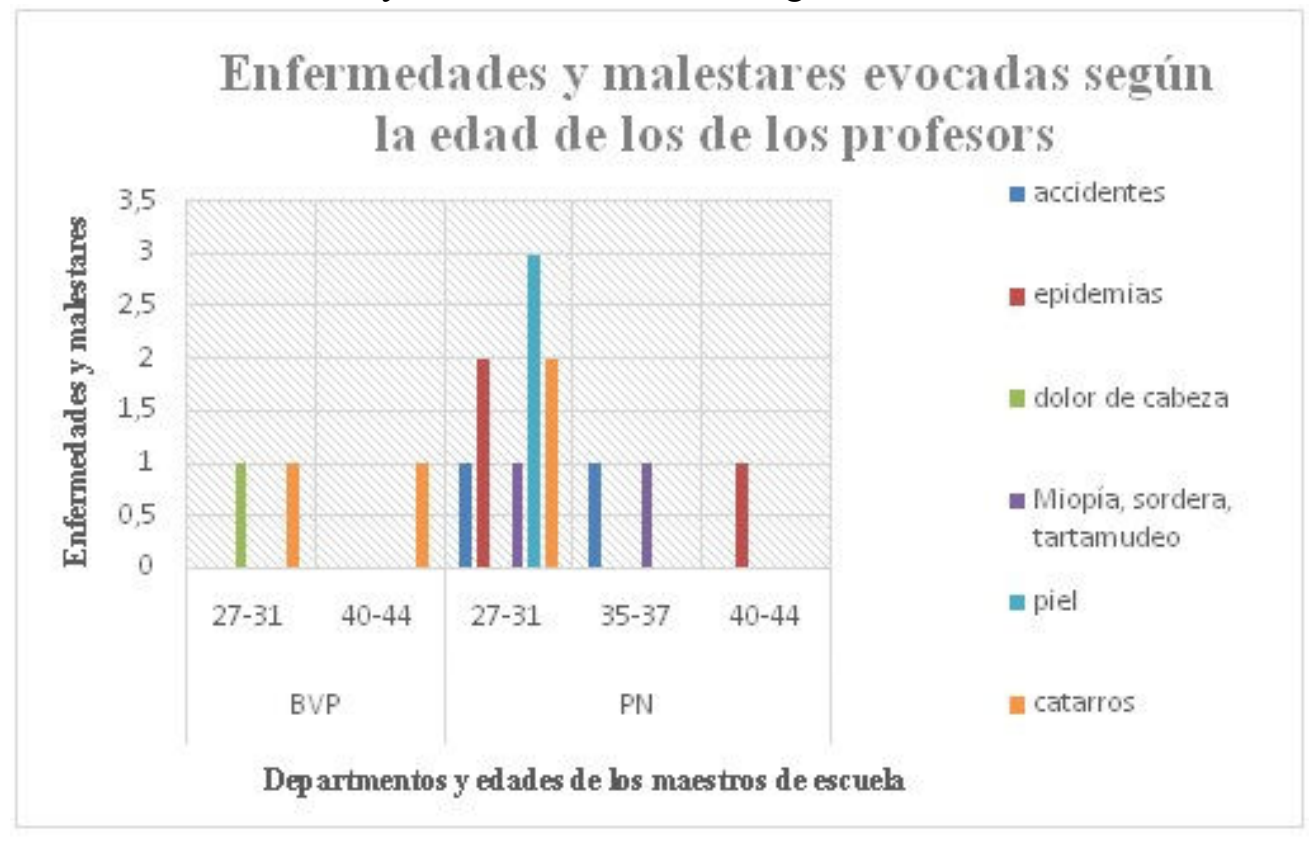

Fuente: Elaboración del autor.

BVP: significa la región de la Bretaña y reagrupa tres departamentos: Finistère, Ile y Vilaine y Morbihan (Noroeste de Francia) n: 108

PN: significa los departamentos del Norte y de Pas-de-Calais (Nordeste de Francia) n: 406

$\mathrm{BVP}+\mathrm{PN}=514$

Pedagogías adecuadas y basadas en la práctica, el ejemplo y la orientación ${ }^{22}$ de las poblaciones

\section{Favorecer los progresos intelectuales para una pedagogía adecuada y práctica}

Algunos maestros entendieron la relación existente entre la asistencia sanitaria y los beneficios aportados por el aprendizaje y el progreso escolar. A semejanza de este profesor del Bas-Rhin (Este de Francia) que expresa cómo "un niño enfermizo no puede asistir regularmente a la escuela, no puede ver exactamente lo que sucede a diario en su clase, se retrasa y por

${ }^{22}$ En el sentido del cuidado de la persona, de su orientación y asistencia. 
consecuencia, su educación se resiente. Esto sigue siendo un punto esencial que debe ser estrictamente observado cuando un profesor quiere ver el progreso en su escuela"23. Para él, la salud no es un elemento secundario, sino primordial en el desarrollo del niño. Ésta ayuda no solamente a su desarrollo físico sino intelectual. El vínculo con la pedagogía se establece, ¿habría entre los profesores, algunas que pudieran adaptarse para favorecer la salud?

El profesor debe también "saber más de lo que enseña" ${ }^{24}$ y ser capaz de adaptarse y documentarse con los avances de su época. Bajo este registro ciertos profesores expresan ideas innovadoras, modernas y procuran adaptar las condiciones de la clase y de las pedagogías a los cuidados del cuerpo. Por ejemplo, modificando los ritmos escolares (son las pausas las que se reivindican), mejorando las condiciones de estudio (sobre todo el material, el mobiliario y variando los ejercicios), mediante la introducción de nuevos métodos de motivación, donde los ejercicios y los juegos tienen un lugar importante para el relajamiento de las enseñanzas consideradas como demasiado estrictas. Este enseñante del Norte propone una verdadera innovación para su época: "Para las escuelas en las que hay miopes, se necesita un pequeño banco para que los libros o los cuadernos se coloquen a cierta distancia de los ojos y aumentar la distancia a medida que el órgano se perfecciona" ${ }^{25}$. Algunos consideran estos métodos como prácticas pedagógicas, lo que constituye de alguna manera el antecedente de "la higiene por el ejemplo"26, que será valorada a finales del siglo XIX (FRIOUX y NOURRISSON, 2015). En Viena y Haute-Vienne (cf. Mapa 1), los maestros insisten en un aprendizaje más práctico orientado para la vida:

Debemos, por tanto, circunscribir las materias que se enseñan en nuestras escuelas con las necesidades y usos, los más ordinarios, los más cercanos a la vida, en vez de proceder por la teoría en la enseñanza como se ha hecho desde hace mucho tiempo y se sigue haciendo en muchas escuelas, hay que llamar la atención de los niños con cuestiones

\footnotetext{
23 "un enfant maladif ne peut suivre régulièrement les cours, il ne peut voir exactement ce qui se fait journellement dans sa classe et il reste en arrière et par conséquent son instruction souffre. C'est donc encore un point essentiel qu'il faut observer strictement quand un instituteur veut voir des progrès dans son école"Arch. Nat. F ${ }^{17}$ 10794, Philippe Molinet, Heiligenberg, Bas-Rhin, 30 janvier 1861.

24 “en savoir plus qu'il n'enseigne”Arch. Nat. F ${ }^{17}$ 10789Jean Monet, Saint-Pierre de Maillé, Vienne, 2 février 1861.

25 "Pour les écoles où il existe des myopes, il faudrait un petit banc afin que les livres ou les cahiers fussent à une certaine distance des yeux et augmenter la distance à mesure que l'organe se perfectionne"Arch. Nat. $\mathrm{F}^{17} 10774$, Pierre-Marie-Auguste Vasseur, Selles, Nord, 25 janvier 1861.

${ }^{26}$ De l'hygiène par l'exemple, existen numerosas referencias pedagógicas a finales del siglo XIX. Una revista será creada con este título en 1921 así como una asociación (fundada en 1920 por Emile Roux, director del Instituto Pasteur de Paris e higienista).
} 
sobre las palabras y las cosas que suceden frente a sus ojos y de esta manera se verán obligados a examinar, a darse cuenta, a resumir y a definir",27.

A este deseo se unió un fuerte reclamo de reivindicación por el aprendizaje práctico de la agricultura y la horticultura, del cultivo de plantas medicinales. Los niños del campo fueron juzgados como más receptivos a este tipo de enseñanza y más necesitados de este aprendizaje en vista de su futura profesión (PARAYRE, 2016).

\section{El profesor: un ejemplo y una guía dentro y fuera de la escuela}

Para los profesores cercanos a su comunidad rural, era menester salir de la escuela y ejercer una influencia saludable entre la población del campo. Este enseñante de Meurthe (Este de France) señala "la negligencia de los habitantes y de la administración local" 28 en materia sanitaria. Con "calles llenas de lodo, aguas estancadas y con estiércol, que han hecho que se extienda un hedor insoportable, nocivo, [...], que fue la causa repetida de enfermedades epidémicas"29 . Por tanto, él "hizo todo lo posible para que quede claro a la autoridad local, de la necesidad y los medios para remediar este deplorable estado de las cosas y nos hemos arreglado para mitigar el daño" ${ }^{30}$. La gráfica 4 muestra las cualidades de un maestro. La dedicación y el hecho de ser una guía eran primordiales. Es importante que el maestro muestre el ejemplo dentro y fuera de clase: "El maestro debe tener una conducta ejemplar, debe evitar lugares insalubres, los cabarets y otros lugares de ocio y de juego, abstenerse de fumar en lugares públicos, mantener al tanto a las autoridades locales de lo que se practica en la escuela"31. En la misma gráfica 4 se comparan varios departamentos, en Corrèze (Sudoeste) y en Bretaña (Nordoeste), los maestros

\footnotetext{
27 "Il faut donc circonscrire les matières enseignées dans nos écoles aux besoins et usages les plus ordinaires, les plus réels de la vie, et au lieu de procéder par la théorie dans l'enseignement comme cela s'est fait pendant longtemps et se fait encore dans un grand nombre d'écoles, il faut appeler l'attention des enfants par des questions sur les mots et les choses qui se passent sous leurs yeux et par ce moyen ils sont forcés à examiner, à se rendre compte, à résumer et à définir" Arch. Nat. $\mathrm{F}^{17} 10789$ André Joseph Lallay, Royères de Saint-Léonard, Haute-Vienne, 30 janvier 1861.

28 "l'incurie des habitants et de l'administration locale"Arch. Nat. F 10783 Louis Léopold Fébvrel, Albestroff, Meurthe, 30 janvier 1860 .

${ }^{29}$ Des "rues remplies de boue, avec des eaux croupissantes et du fumier, ont répandu une odeur infecte insupportable, délétère, [...] qui a été la cause à plusieurs reprises de maladies épidémiques" Ibid.

30 "fait son possible pour faire comprendre à l'autorité locale la nécessité et les moyens de remédier à cet état de choses déplorable et l'on est parvenu à atténuer le mal"Ibid.

31 "L'instituteur doit avoir une conduite exemplaire, il doit éviter de fréquenter des lieux malsains, les cabarets et autres lieux de divertissements et de jeux, s'abstenir de fumer dans les lieux publics, tenir les autorités locales au courant de ce qui se pratique à l'école"Arch. Nat. F ${ }^{17} 10783$ Jean-Baptiste Renaud, Bébing, Meurthe, 24 janvier 1861.
} 
hablan poco de las cualidades personales que se requieren para ejercer la profesión. Son aquellos que parecen los mas desinteresados en la salud y en la educación corporal. Por el contrario, son los profesores más implicados en las áreas de la salud y la educación corporal, los que revelan sus cualidades y reivindican la obligación de contar con cualidades por demás ejemplares para poder ejercer a cabalidad todas las dimensiones de la educación.

Gráfica 4: Cualidades del profesor.

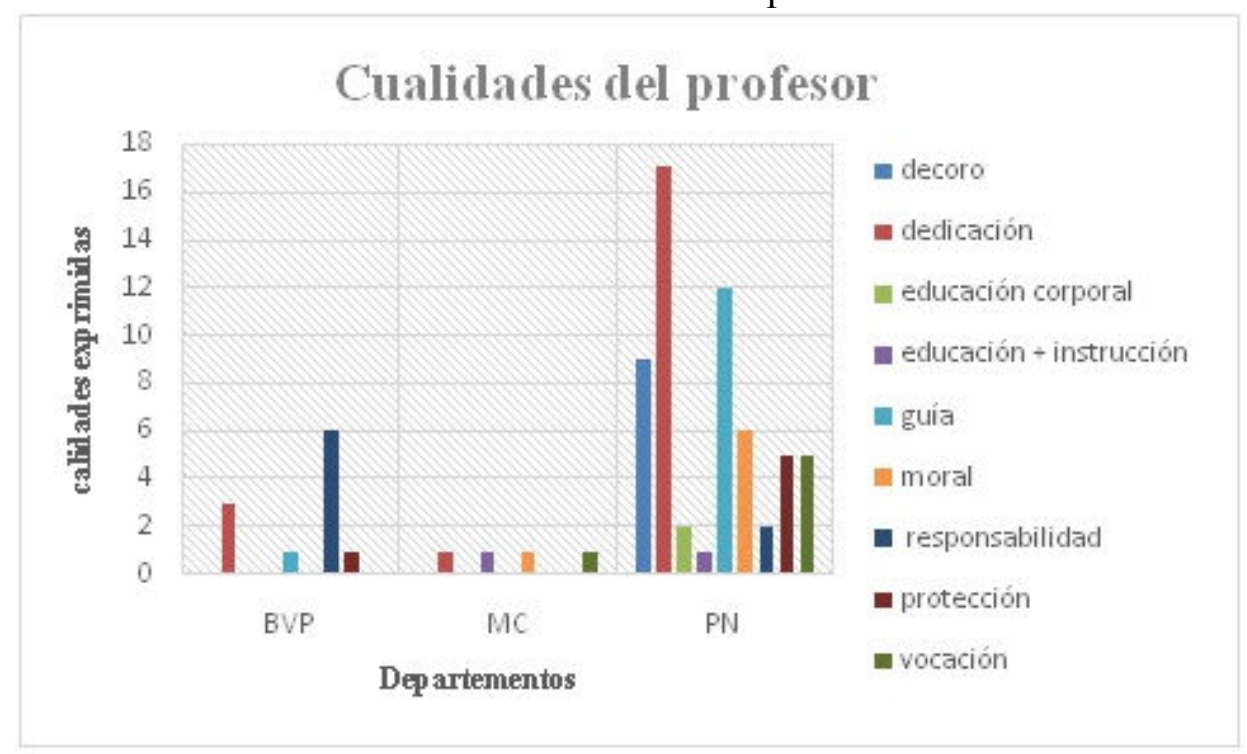

Fuente: Elaboración del autor.

BVP: significa la región de la Bretaña y reagrupa tres departamentos: Finistère, Ile y Vilaine et Morbihan (Noroeste de France) n: 108

PN: significa les departamentos del Norte y de Pas-de-Calais (Nordeste de France) n: 406

MC: significa departamento de la Corrèze (Sudoeste de France) n: 53

$\mathrm{BVP}+\mathrm{PN}+\mathrm{MC}=567$

\section{DISCUSIÓN-CONCLUSIÓN}

Después de un primer análisis de este corpus, inédito y rico, sobre los discursos de los profesores, quizá el primero en proporcionar tanta información sobre las opiniones de los maestros rurales; no podemos generalizar sobre los hallazgos en torno al cuidado de la salud y el cuerpo en el conjunto de la profesión docente. Los escasos resultados y las gráficas muestran que un puñado confiaba en las memorias su opinión, orientación y prescripción sobre aquellos elementos del cuerpo y la salud, pero aún resultan marginales (si lo comparamos con el conjunto de la profesión y la variedad de temas abordados). Este estudio no determina tampoco cuáles 
podrían ser las acciones sobre la salud en el terreno escolar. Podemos suponer que quienes las evocan parecen lo suficientemente sinceros para no encubrir sus propósitos haciéndose pasar como profesores modelo ante sus superiores. Los comentarios negativos señalados por los inspectores de academia que rechazaron estas memorias demuestran por el contrario, que no son ni la salud ni la higiene y tampoco la educación corporal los que podrían aportar puntos suplementarios para remontar en la clasificación. Es de notarse además el esfuerzo suplementario para escribir las memorias visto que su carga de trabajo ${ }^{32}$ no les permitía destinar mucho tiempo a la escritura.

Sin embargo, esta investigación tiene su originalidad por ser una "historia desde abajo" vista desde los discursos de los maestros y no sólo de los médicos, administradores o las élites. La mayoría de los profesores proviene de zonas rurales y quieren educar y apoyar a la población rural (PARAYRE, 2016). En este registro, el corpus muestra que algunos de ellos son capaces de innovar y sobresalir, sin dudar siquiera en sustituir al médico si es necesario. También es interesante ver que las referencias a la medicina científica son muchas, aunque algunos otros se refieran a fuentes y a experiencias, que atienden más a su intuición y a su entendimiento sobre la salud, lo que los investigadores definen hoy como un "savoir expérientiel en santé" (JOUET, LAS VERGNAS, NOEL-HUREAUX, 2014, p. 63; POLS, 2014).

En el contexto de la época, este fenómeno parece bastante obvio, los maestros no han recibido ningún tipo de formación específica en materia de higiene y se refieren todavía al conocimiento profano. No se han sometido, ni se encuentran imbuidos de alguna manera, por la biomedicalización de la sociedad. Será interesante conocer si los maestros de la Tercera República (1870-1940) seguirán las mismas referencias y se adherirán a los mismos mensajes que se transmiten por la cultura higienista y pasteuriana, de alguna manera, si una interiorización de biomedicalización, terminará por influirlos en sus prácticas pedagógicas y escolares (DELUERMOZ, 2012).

Otro elemento relevante, que resulta totalmente pertinente, es el espíritu innovador de algunos de ellos, modernos en su pedagogía y en las acciones propuestas, en la dedicación que comparten. Es este elemento el que nos lleva a querer profundizar aún más el estudio. Porque este corpus es sólo una primera parte de una historia de la educación para la salud, y debe

\footnotetext{
${ }^{32}$ Recordemos que en esta época había muchos niños en el salón de clases, a veces 80 y hasta 100 alumnos a cargo de un solo maestro quien podía tener además la función de secretario del ayuntamiento o alguna otra obligación eclesiástica en la parroquia
} 
confrontarse con otras fuentes para reconstruir los caminos que toman las variadas actitudes. Así podremos aprehender diferentes aspectos de esta historia que no podría resumirse en un sólo modelo de biomedicalización (FAURE, 2015). Puede ser que se nos reproche de no hablar de maestros del campo. Esta parte de la investigación está por venir, es inherente a las fuentes disponibles, pues por el momento no hemos encontrado un corpus similar tan rico sobre los maestros del medio rural. Es una investigación que puede llevarnos a observar las pedagogías empleadas y reivindicadas por los maestros y sus opiniones sobre la salud de los alumnos, si difieren, sobre qué elementos y por qué razones.

El corpus estudiado que se sitúa en el límite de la Tercera República (1870) y su gran empresa de aculturación de la biomedicalización de la sociedad, muestra que veinte años antes de las Leyes Ferry (1881-1882) sobre la obligación, la gratuidad y la laicidad de la escuela; los maestros rurales reivindican estos avances. Lo mismo para la salud, la higiene y el cuerpo: una veintena de años antes de la obligatoriedad en las escuelas, existen enseñanzas específicas como la higiene, el desarrollo de la gimnastica y de su enseñanza obligatoria (leyes Ferry de 1882); los maestros rurales son sensibles, reivindican y aplican desde entonces los principios de cuidado ligados al desarrollo del niño en su integralidad. No esperaron una indicación o exigencia del Ministerio para hacerlo. La existencia de actitudes similares (de maestros que no esperan a la directiva, ni una obligación para reaccionar) podrá ser investigada a profundidad en las proximas investigaciones.

Estos pasajes sobre la salud, sus cuidados y prevención presentan varias imágenes de la escuela y del sistema educativo, que evolucionaran en el curso de la Tercera República (18701940). La escuela es vista, en principio, como un lugar que no debe deteriorar la salud y, por tanto, debe procurar el cuidado de los estudiantes, desde la ventilación de los locales hasta el acondicionamiento de los centros de salud. La escuela es un lugar de vida que debe respetar el desarrollo del niño y puede prevenir la propagación de enfermedades y males. El médico Riant especialista de la higiene lo señala en su libro Hygiène scolaire, influence de l'école sur la santé des enfants (RIANT, 1874). Durante el siglo XIX se añaden otros elementos nocivos que también afectan a la pedagogía, los aprendizajes puede ser perjudiciales para la salud y causar una serie de inconvenientes, o incluso deformaciones, mala postura, problemas de visión, de voz, etc. Se entra en la era en la que la escuela puede estar en el origen de enfermedades y malestares, es la escuela que deteriora la salud. La escuela es también el lugar privilegiado de las normas y los estándares. 
La higiene y su vasta extensión durante la Tercera República (1870-1940) propaga una serie de normas y comportamientos sanitarios y contará con los maestros de escuela primaria, hombres y mujeres, quienes serán los promotores principales de la medicalización de la población. También será viable buscar otras facetas de la escuela, a veces contradictorias, a veces complementarias, en las que un conjunto de valores, actitudes, umbrales de diferentes sensibilidades, pueda estar en el origen de otras observaciones de la infancia. Considerarla como una concepción dinámica en el que un mismo modelo pueda dominar (que parece ser el caso del higienismo), pero también pueda ser criticado, depuesto y reemplazado (lo que también sería el caso higienismo) ${ }^{33}$.

\section{BIBLIOGRAFÍA}

ARCHIVOS NACIONALES DE PARÍS. Francia. $F^{17}$ 10758-10798: concours ouvert aux instituteurs, 1861.

BOURDELAIS, Patrice. Les logiques du développement de l'hygiène publique. Dans Bourdelais Patrice (dir.). Les hygiénistes, enjeux, modèles et pratiques. Paris: Belin, 2001, 5-26.

CARON, François. Histoire des chemins de fer en France (tome 1, 1740-1883, tome 2, 18831937). Paris: Fayard, 1997 et 2005.

CHEVALIER, Louis. Classes laborieuses et classes dangereuses. Paris: Perrin, 2007.

DELUERMOZ, Quentin (dir.). Norbert Elias. Paris: Tempus Perrin, 2012.

FAURE, Olivier. Aux marges de la médecine: santé et souci de soi, France (XIXe siècle). Publications de L'Université de Provence, 2015.

FURET, François; OZOUF, Jacques. Lire et Écrire, l'alphabétisation des Français de Calvin à Jules Ferry. Paris: Éditions de Minuit, 2 vols, 1977.

FRIOUX, Stéphane; NOURRISSON, Didier. Propre et sain! Un siècle d'hygiène à l'école en images. Paris: Armand Colin, 2015.

GHERARDI, Eugène F.-X. Être instituteur en Corse sous le Second Empire. Ajaccio: Albiana, Università di Corsica, coll. "Bibliothèque de la Corse", 2012.

\footnotetext{
33 Un agradecimiento especial a la profesora Lucía Martínez Moctezuma por su enriquecedora colaboración, por compartir conmigo el desafío de esta propuesta y aceptar traducir este artículo al español que permitirá ampliar la difusión de mis trabajos de investigación. Agradezco también a Denise Bernuzzi de Sant'Anna por la traducción del resumen en portugués.
} 
GRÉARD, Octave. La Législation de l'instruction primaire en France depuis 1789 jusqu'à nos jours, recueil des lois, décrets, ordonnances, arrêtés, règlements, décisions, avis, projets de lois. Paris: Delalain frères, t. III, de 1848 à 1863.

JACQUET-FRANCILLON, François. Instituteurs avant la République. La profession d'instituteur et ses représentations de la monarchie de Juillet au Second Empire. Villeneuve d'Ascq: Presses Universitaires du Septentrion, 1999.

JOUET, Emmanuelle; LAS VERGNAS, Olivier; NOEL-HUREAUX, Elisabeth. Nouvelles coopérations réflexives en santé: De l'expérience des malades et des professionnels, aux partenariats de soins, de formation et de recherche. Paris: Editions des archives contemporaines, 2014.

JOURNAL DES INSTITUTEURS, nº 38 du 22 septembre 1861.

KLEIN, Alexandre; PARAYRE, Séverine (dir.). Histoire de la santé XVIIIe-XXe siècles. Nouvelles recherches francophones. Laval: Presses Universitaires de Laval, 2015.

LEONARD, Jacques. La France médicale, médecins et malades au XIXe siècle. Paris: Gallimard., 1978.

La médecine entre les savoirs et les pouvoirs, histoire intellectuelle et politique de la médecine française au XIXe siècle. Paris: Aubier Montaigne, 1981.

LORRAIN, Paul. Tableau de l'instruction primaire en France d'après les rapports adressés au ministre de l'Instruction publique par les 490 inspecteurs chargés de visiter toutes les écoles de France, à la fin de 1833. Paris: L. Hachette, 1837.

MANGENOT, Charles. L'inspection hygiénique et médicale des écoles, extrait de la Revue d'hygiène et de police sanitaire. Paris, G. Masson, 1887.

MARISSAL, Claudine. Protéger le jeune enfant, enjeux sociaux, politiques et sexués, Belgique, 1890-1940. Bruxelles: Editions de l'Université de Bruxelles, 2014.

NICOLAS, Gilbert. Quand les instituteurs répondaient au ministre: mémoires des maîtres de l'enseignement primaire sous le Second Empire. Rennes: Presses universitaires de Rennes, 2012.

NOURRISSON, Didier (dir.). Education à la santé XIXe-XXe siècle. Rennes: Éd. de l'école nationale de la santé publique, 2002.

OZOUF, Jacques. Nous les maîtres d'école, autobiographies d'instituteurs de la belle époque. Paris: Folio histoire, 1999.

OZOUF, Mona. La classe ininterrompue, cahiers de la famille Sandre, enseignants 17801960. Paris: Hachette, 1979. 
PARAYRE, Séverine. Les prémices de l'éducation à la santé au XIXe siècle: implication et formation des maîtres. Dans BERGER Dominique (dir.), Education à la santé: enjeux et dispositifs à l'école. Toulouse: Editions universitaires du Sud, 2010, 331-349.

. L'hygiène à l'école, une alliance de la santé et de l'éducation XVIIIe-XIXe siècles. Saint-Etienne: PUSE, 2011.

. Valorisation de l'agriculture à l'école primaire, Éduquer, former et socialiser les futurs paysans en 1861. Dans MORICEAU Jean.-Marc et MADELINE Philippe. Les petites gens de la terre. Caen: Bibliothèque du Pôle Rural (BPR 14), 2016.

. The internationalization of school hygiene between the hegemony of European model and local innovations of medicalization (XIXth-XXth centuries). Canadian Bulletin of Medical History. Toronto: University of Toronto Press, 2017.

POLS, Jeannette. Knowing Patients: Turning Patient Knowledge into Science. Science, Technology, \& Human Values, 39(1), 73-97, 2014. Disponible en: $<$ http://sth.sagepub.com/content/39/1/73.full.pdf + html $>$.

PÉLISSIER, Jean.-Pierre; RÉBAUDO Danièle. Une approche de l'illettrisme en France, la signature des actes de mariage au xIxe siècle dans l'enquête 3000 familles. Histoire et Mesure, IX, 1/2, 161-202, 2004.

PORTER, Roy. The Greatest Benefit to Mankind. A Medical History of Humanity. New York, London: W.W. Norton \& Compagny, 1997.

ROBERT, Charles. Plaintes et vœux présentés par les instituteurs publics en 1861 sur la situation des maisons d'école, du mobilier et du matériel classiques. Paris, De Guillaumin et Cie, 1864.

RIANT, Aimé. Hygiène scolaire, influence de l'école sur la santé des enfants. Paris: Hachette, 1874.

RIONDET, Xavier; GO, Henri.- Louis. Freinet et les phobies scolaires: une conception écologique des problèmes. La Nouvelle Revue de l'Adaptation et de la Scolarisation, 62, 2333, 2013.

De l'expérience de la tuberculose aux pratiques de santé d'Elise Freinet. Eléments pour comprendre une éducation à la santé. Dans Klein Alexandre et PARAYRE Séverine (dir.), Histoire de la santé XVIIIe-XXe siècles, nouvelles recherches francophones. Laval: Presses Universitaires de Laval, 2015, p.81-101.

VIGARELLO, Georges. Le corps redressé, histoire d'un pouvoir pédagogique. Paris: Armand Colin, 2004, 1re éd. 1978.

. Le sentiment de soi. Histoire de la perception du corps. Paris: Seuil, 2014. 